\title{
QUADRATIC HYPERBOLICITY PRESERVERS AND MULTIPLIER SEQUENCES
}

\author{
R. BATES AND R. YOSHIDA
}

\begin{abstract}
It is known (see [4, Brändén, Lemma 2.7]) that a necessary condition for $T:=\sum Q_{k}(x) D^{k}$ to be hyperbolicity preserving is that $Q_{k}(x)$ and $Q_{k-1}(x)$ have interlacing zeros. We characterize all quadratic linear operators; as a consequence, we find several classes of the $P_{n}$-multiplier sequence.
\end{abstract}

1. Introduction. It is well known (see [8], [9, page 32]) that, if $T$ is any linear operator defined on the space of real polynomials, $\mathbb{R}[x]$, then there is a sequence of real of polynomials, $\left\{Q_{k}(x)\right\}$, such that

$$
T=\sum Q_{k}(x) D^{k}, \quad \text { where } D=\frac{d}{d x} .
$$

Our investigation involves such operators that act on polynomials, in particular, we are interested in polynomials with the following property.

Definition 1.1. A polynomial $f(x) \in \mathbb{R}[x]$ whose zeros are all real is said to be hyperbolic. Following the convention of Pólya and Schur [10, page 89], the constant 0 is also deemed to be hyperbolic.

Definition 1.2. A linear operator $T: \mathbb{R}[x] \rightarrow \mathbb{R}[x]$ is said to be preserve hyperbolicity (or $T$ is a hyperbolicity preserver) if $T[f(x)]$ is a hyperbolic polynomial, whenever $f(x)$ is a hyperbolic polynomial.

Hyperbolicity preserving operators have been studied by virtually every author who has studied hyperbolic polynomials (see [5] and the references contained therein). The focus of our investigation

2010 AMS Mathematics subject classification. Primary 33C47, 26C10, Secondary 30C15, 33C52.

Keywords and phrases. Laguerre-Pólya class, multiplier sequences, linear operators.

Received by the editors on June 5, 2013, and in revised form on April 12, 2014. 
involves the relationship between hyperbolicity preserving operators and hyperbolic polynomials with interlacing zeros.

Definition 1.3. Let $f, g \in \mathbb{R}[x]$ with $\operatorname{deg}(f)=n$ and $\operatorname{deg}(g)=m$. We say that $f$ and $g$ have interlacing zeros, if $f$ is hyperbolic with zeros $\alpha_{1}, \ldots, \alpha_{n}$ and $g$ is hyperbolic with zeros $\beta_{1}, \ldots, \beta_{m}$, where $|n-m| \leq 1$, with one of the following forms holding:

(i) $\alpha_{1} \leq \beta_{1} \leq \alpha_{2} \leq \beta_{2} \leq \ldots \leq \alpha_{n} \leq \beta_{m}$,

(ii) $\beta_{1} \leq \alpha_{1} \leq \beta_{2} \leq \alpha_{2} \leq \ldots \leq \beta_{m} \leq \alpha_{n}$,

(iii) $\alpha_{1} \leq \beta_{1} \leq \alpha_{2} \leq \beta_{2} \leq \ldots \leq \beta_{m} \leq \alpha_{n}$, or

(iv) $\beta_{1} \leq \alpha_{1} \leq \beta_{2} \leq \alpha_{2} \leq \ldots \leq \alpha_{n} \leq \beta_{m}$.

We will also say that the zeros of any two hyperbolic polynomials of degree 0 or 1 interlace. By convention, the zero polynomial interlaces with any hyperbolic polynomial.

Definition 1.4. Given two non-zero polynomials $f, g \in \mathbb{R}[x]$, we say $f$ and $g$ are in proper position and write $f \ll g$ if one of the following conditions holds:

(i) $f$ and $g$ have interlacing zeros with form (i) or (iv) in Definition 1.3, and the leading coefficients of $f$ and $g$ are of the same sign, or

(ii) $f$ and $g$ have interlacing zeros with form (ii) or (iii) in Definition 1.3, and the leading coefficients of $f$ and $g$ are of opposite sign.

We will say that the zero polynomial is in proper position with any other hyperbolic polynomial $f$ and write $0 \ll f$ or $f \ll 0$.

Notice that, by Definition 1.4, if $f$ and $g$ are in proper position, then $f$ and $g$ are hyperbolic. Also, to be clear, a non-zero constant can only be in proper position with another constant or a linear polynomial. However, the zero polynomial is in proper position with any hyperbolic polynomial.

Definition 1.5. For any two real polynomials $f$ and $g$, the Wronskian of $f$ and $g$ is defined, on $\mathbb{R}$, by

$$
W[f, g]:=f(x) g^{\prime}(x)-f^{\prime}(x) g(x) .
$$


It is a common exercise to show that, for $f$ and $g$ with interlacing zeros, if $W[g, f] \leq 0$ on the whole real line, then $f \ll g$.

The following lemma demonstrates that proper position plays an important role in understanding hyperbolicity preservers.

Lemma 1.6. [4, Lemma 2.7]. Suppose the linear operator

$$
T=\sum_{k=M}^{N} Q_{k}(x) D^{k},
$$

where $Q_{k}(x) \in \mathbb{R}[x]$ for $M \leq k \leq N$, and $Q_{M}(x) Q_{N}(x) \not \equiv 0$, preserves hyperbolicity. Then $Q_{j}(x) \ll Q_{j+1}(x)$ for $M \leq j \leq N-1$. In particular, $Q_{j}(x)$ is hyperbolic or identically zero for all $M \leq j \leq N$.

In the special case for $M=0$ and $N=2$ in (1.2), we find sufficient conditions that guarantee when $T$ preserves hyperbolicity. Our main result is the following:

Theorem 1.7. Suppose $Q_{2}, Q_{1}, Q_{0}$ are real polynomials such that $\operatorname{deg}\left(Q_{2}\right)=2, \operatorname{deg}\left(Q_{1}\right) \leq 1, \operatorname{deg}\left(Q_{0}\right)=0$. Then

$$
T=Q_{2} D^{2}+Q_{1} D+Q_{0}
$$

preserves hyperbolicity if and only if

$$
W\left[Q_{0}, Q_{2}\right]^{2}-W\left[Q_{0}, Q_{1}\right] W\left[Q_{1}, Q_{2}\right] \leq 0, \quad Q_{0} \ll Q_{1} \text { and } Q_{1} \ll Q_{2} .
$$

2. Quadratic hyperbolicity preservers. We concern ourselves with operators of the following form.

Definition 2.1. We will call the second order differential operators of the form

$$
T=Q(x) D^{2}+P(x) D+R(x)
$$

a quadratic operator, where the polynomials: $Q(x)$ is quadratic, $P(x)$ is linear and $R(x)$ is constant. If (2.1) is also hyperbolicity preserving, then we will refer to it as a quadratic hyperbolicity preserver. 
The following proposition presents an operator that has been quite influential to our exposition.

Proposition 2.2 (Forgács et al. [1, Proposition 5]). If $0<d<1$, then the operator

$$
T=\left(x^{2}-1\right) D+2 x D+d
$$

preserves hyperbolicity.

For motivation, we present several other similar examples of quadratic operators.

\section{Example 2.3.}

$$
\begin{aligned}
& T_{1}=\left(x^{2}-1\right) D^{2}+2 x D-1 \\
& T_{2}=\left(x^{2}-1\right) D^{2}+2 x D+0 \\
& T_{3}=\left(x^{2}-1\right) D^{2}+2 x D+1 \\
& T_{4}=\left(x^{2}-1\right) D^{2}+2 x D+2 \\
& T_{5}=\left(x^{2}-1\right) D^{2}-2 x D-1 \\
& T_{6}=\left(x^{2}-1\right) D^{2}-2 x D+0 \\
& T_{7}=\left(x^{2}-1\right) D^{2}-2 x D+1 \\
& T_{8}=\left(x^{2}-1\right) D^{2}-2 x D+2
\end{aligned}
$$

It was shown in [1, Lemma 5] that (2.4) is hyperbolicity preserving. Notice that $T_{2}=D\left(x^{2}-1\right) D$, and thus (2.3), is hyperbolicity preserving as well. The other six examples can easily be shown not to preserve hyperbolicity.

$$
\begin{aligned}
T_{1}\left[x^{2}-1\right] & =5 x^{2}+2 . \\
T_{4}\left[(x-10)^{3}\right] & =2(x-10)\left(7 x^{2}-50 x+97\right) . \\
T_{5}\left[x^{2}\right] & =-3 x^{2}-2 . \\
T_{6}\left[x^{2}\right] & =-2 x^{2}-2 . \\
T_{7}\left[x^{2}\right] & =-x^{2}-2 . \\
T_{8}\left[(x-10)^{3}\right] & =2(x-10)\left(x^{2}+10 x+97\right) .
\end{aligned}
$$


These examples show that the property of interlacing coefficients is not sufficient to establish hyperbolicity preserving. Furthermore, (2.5) demonstrates that the condition of proper position in Lemma 1.6 is also not sufficient to establish hyperbolicity preserving. The examples motivate us to find the necessary and sufficient conditions on the polynomial coefficients in the quadratic operator (2.1).

We will completely characterize all quadratic hyperbolicity preservers. For our characterization, we will need a result due to Borcea and Brändén.

Theorem 2.4 (Borcea and Brändén [3, Theorem 1.3]). Let $T: \mathbb{R}[x] \rightarrow$ $\mathbb{R}[x]$ be a finite-differential linear operator such that, for some real polynomials $\left\{Q_{k}(x)\right\}_{k=0}^{n}$,

$$
T=\sum_{k=0}^{n} Q_{k}(x) D^{k} .
$$

Then $T$ is hyperbolicity preserving if and only if

$$
\sum_{k=0}^{n} Q_{k}(x)(-w)^{k} \neq 0
$$

for every $x, w \in H^{+}$, where $H^{+}$is the upper half plane.

In general, Theorem 2.4 can be difficult to apply since very little is known about two variable stable polynomials (see [3]). The next few lemmas establish a small class of two variable stable polynomials.

Lemma 2.5. Let $A, B \in \mathbb{C}-\mathbb{R}$ be two non-real complex numbers such that

(i) $0<\operatorname{Arg}(B)<\operatorname{Arg}(A)<2 \pi$,

(ii) $\operatorname{Arg}(A)-\operatorname{Arg}(B)<\pi$, and

(iii) $\Im(A)<\Im(B)$.

Then, for any $r_{1}, r_{2} \in \mathbb{R}, r_{1}<r_{2}$, there exist $x, w \in H^{+}$such that $\left(x+r_{1}\right) w=A$ and $\left(x+r_{2}\right) w=B$.

Proof. Consider the following cases. 
Case 1. $B \in H^{+}$. The point $B$ may be located in either quadrant I, on the positive imaginary axis, or in quadrant II, as described in Figure 1. The hypotheses (i), (ii) and (iii) imply that point $A$ is located somewhere in the shaded region of the corresponding point $B$.
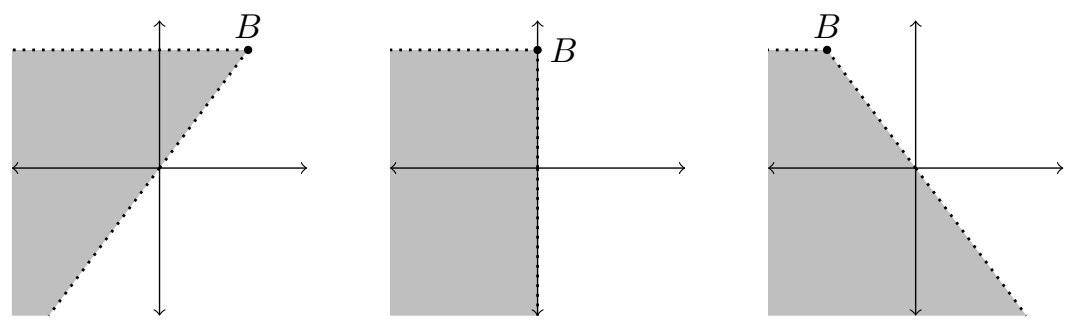

FIGURE 1

Define the function $f:[0, \operatorname{Arg}(B)] \rightarrow \mathbb{R}$ by

$$
f(\theta):=\Im\left(e^{-i \theta} A\right)-\Im\left(e^{-i \theta} B\right) .
$$

Then $f(0)<0$ by (iii), and $f(\operatorname{Arg}(B))>0$ by (ii). Thus, by continuity, there exist $\theta_{0} \in(0, \operatorname{Arg}(B))$ such that $f\left(\theta_{0}\right)=0$, which implies that $\left(e^{-i \theta_{0}} B-e^{-i \theta_{0}} A\right)>0$ by (i). Define the function $g:[0, \infty) \rightarrow \mathbb{R}$ by

$$
g(k):=k\left(e^{-i \theta_{0}} B-e^{-i \theta_{0}} A\right) .
$$

Notice $g \geq 0, g(0)=0$, and $\lim _{k \rightarrow+\infty} g(k)=+\infty$. Thus, there exists $k_{0}>0$ such that $g\left(k_{0}\right)=r_{2}-r_{1}$. Let

$$
x=\frac{1}{2}\left(k_{0} e^{-i \theta_{0}} B+k_{0} e^{-i \theta_{0}} A-r_{1}-r_{2}\right) \quad \text { and } \quad w=\frac{1}{k_{0}} e^{i \theta_{0}} .
$$

It follows that $x, w \in H^{+},\left(x+r_{1}\right) w=A$, and $\left(x+r_{2}\right) w=B$.

Case 2. $B \in H^{-}$. Similar to Case 1, point $B$ may be located in either quadrant III, on the negative imaginary axis, or in quadrant IV, as described in Figure 2. Point $A$ is located somewhere in the shaded region of the corresponding point $B$ by hypotheses (i), (ii) and (iii).

Define the function $f:[0,2 \pi-\operatorname{Arg}(B)] \rightarrow \mathbb{R}$ by

$$
f(\theta):=\Im\left(e^{i \theta} A\right)-\Im\left(e^{i \theta} B\right) .
$$

Then $f(0)<0$ by (iii), and $f(2 \pi-\operatorname{Arg}(B))>0$ by (ii). Thus, by continuity, there exist $\theta_{0} \in(0,2 \pi-\operatorname{Arg}(B))$ such that $f\left(\theta_{0}\right)=0$, 

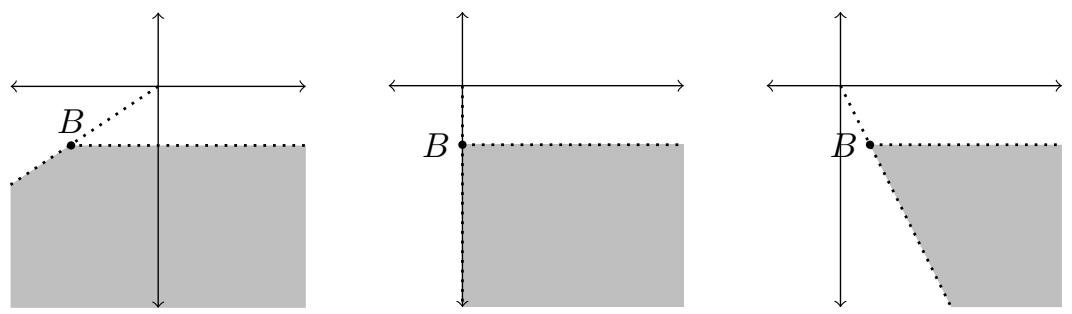

Figure 2

which implies that $\left(e^{i \theta_{0}} B-e^{i \theta_{0}} A\right)<0$ by (i). Define the function $g:(-\infty, 0] \rightarrow \mathbb{R}$ by

$$
g(k):=k\left(e^{i \theta_{0}} B-e^{i \theta_{0}} A\right) .
$$

Then $g \geq 0, g(0)=0$, and $\lim _{k \rightarrow-\infty} g(k)=+\infty$. Thus, there exist $k_{0}<0$ such that $g\left(k_{0}\right)=r_{2}-r_{1}$. Let

$$
x=\frac{1}{2}\left(k_{0} e^{i \theta_{0}} B+k_{0} e^{i \theta_{0}} A-r_{1}-r_{2}\right), \quad \text { and } \quad w=\frac{1}{k_{0}} e^{-i \theta_{0}} .
$$

It follows that $x, w \in H^{+},\left(x+r_{1}\right) w=A$ and $\left(x+r_{2}\right) w=B$.

Lemma 2.6. Let $a, b, r_{1}, r_{2}, r \in \mathbb{R}, a, b \geq 0$, and $r_{1} \neq r_{2}$. Set

$$
f(x, w)=\left(\left(x+r_{1}\right) w-a\right)\left(\left(x+r_{2}\right) w-b\right), \quad x, w \in \mathbb{C} .
$$

Then

$$
\begin{gathered}
f(x, w) \neq r \quad \text { for all } x, w \in H^{+} \\
\text {if and only if } r \in[0, a b] .
\end{gathered}
$$

Proof. Since the factors of $f(x, w)$ in $(2.22)$ are symmetric, we let $r_{1}<r_{2}$. There are three cases to prove necessity. The following is the outline.

Case 1. $r \in(-\infty, 0)$ and $a<b+2 \sqrt{|r|}$.

Case 2. $r \in(-\infty, 0)$ and $a \geq b+2 \sqrt{|r|}$.

Case 3. $r \in(a b, \infty)$.

We show in each case that there exist $x, w \in H^{+}$such that $f(x, w)=r$. 
Case 1. Consider $r \in(-\infty, 0)$ and $a<b+2 \sqrt{|r|}$. Define $g:[0, \pi / 2] \rightarrow \mathbb{R}$ by

$$
\begin{aligned}
g(\theta) & :=\left(\sqrt{|r|} e^{i \theta}+b\right)-\left(\sqrt{|r|} e^{i(\pi-\theta)}+a\right) \\
& =\sqrt{|r|}(2 \cos (\theta))-a+b .
\end{aligned}
$$

The function $g$ is real valued and $g(0)=b+2 \sqrt{|r|}-a>0$ by assumption. Thus, by continuity, there exists $\theta_{0} \in(0, \pi / 2)$ such that $g\left(\theta_{0}\right)>0$, which implies the following.

(a) $\Im\left(\sqrt{|r|} e^{i \theta_{0}}+b\right)-\Im\left(\sqrt{|r|} e^{i\left(\pi-\theta_{0}\right)}+a\right)=0$,

(b) $\Re\left(\sqrt{|r|} e^{i \theta_{0}}+b\right)-\Re\left(\sqrt{|r|} e^{i\left(\pi-\theta_{0}\right)}+a\right)>0$, and

(c) $\left(\sqrt{|r|} e^{i \theta_{0}}+b\right),\left(\sqrt{|r|} e^{i\left(\pi-\theta_{0}\right)}+a\right) \in H^{+}$.

By (a), (b) and (c),

$$
\operatorname{Arg}\left(\sqrt{|r|} e^{i\left(\pi-\theta_{0}\right)}+a\right)-\operatorname{Arg}\left(\sqrt{|r|} e^{i \theta_{0}}+b\right)>0 .
$$

Define the function $h:(0,1] \rightarrow \mathbb{R}$ by

$$
h(k):=\operatorname{Arg}\left(k \sqrt{|r|} e^{i\left(\pi-\theta_{0}\right)}+a\right)-\operatorname{Arg}\left(\frac{\sqrt{|r|}}{k} e^{i \theta_{0}}+b\right) .
$$

The function $h$ is real valued, and $h(1)>0$. Thus by continuity, there exists $k_{0} \in(0,1)$ such that $h\left(k_{0}\right)$ remains positive,

$$
\operatorname{Arg}\left(k_{0} \sqrt{|r|} e^{i\left(\pi-\theta_{0}\right)}+a\right)-\operatorname{Arg}\left(\frac{\sqrt{|r|}}{k_{0}} e^{i \theta_{0}}+b\right)>0,
$$

but the imaginary parts differ,

$$
\Im\left(k_{0} \sqrt{|r|} e^{i\left(\pi-\theta_{0}\right)}+a\right)<\Im\left(\frac{\sqrt{|r|}}{k_{0}} e^{i \theta_{0}}+b\right) .
$$

Let

$$
A=k_{0} \sqrt{|r|} e^{i\left(\pi-\theta_{0}\right)}+a, \quad \text { and } \quad B=\frac{\sqrt{|r|}}{k_{0}} e^{i \theta_{0}}+b .
$$

Then (2.26) and (2.27) satisfy items (i), (ii) and (iii) of Lemma 2.5; hence, there exist $x, w \in H^{+}$such that $\left(x+r_{1}\right) w=A$ and $\left(x+r_{2}\right) w=$ 
$B$. Thus,

$$
\begin{aligned}
f(x, w) & =\left(\left(x+r_{1}\right) w-a\right)\left(\left(x+r_{2}\right) w-b\right) \\
& =\left(k_{0} \sqrt{|r|} e^{i\left(\pi-\theta_{0}\right)}\right)\left(\frac{\sqrt{|r|}}{k_{0}} e^{i \theta_{0}}\right)=-|r|=r .
\end{aligned}
$$

Case 2. We consider $r \in(-\infty, 0)$ and $b+2 \sqrt{|r|} \leq a$. We will only need $b<a+2 \sqrt{|r|}$. This is easily seen to be true by adding $2 \sqrt{|r|}$ to both sides of $b+2 \sqrt{|r|} \leq a$ and observing $b<b+4 \sqrt{|r|}$. Define the function $g:[0, \pi / 2] \rightarrow \mathbb{R}$ by

$$
\begin{aligned}
g(\theta) & :=\left(\sqrt{|r|} e^{i(2 \pi-\theta)}+a\right)-\left(\sqrt{|r|} e^{i(\pi+\theta)}+b\right) \\
& =\sqrt{|r|}(2 \cos (\theta))+a-b .
\end{aligned}
$$

Again, $g$ is real valued, and $g(0)=a+2 \sqrt{|r|}-b>0$. Thus, by continuity, there exists $\theta_{0} \in(0, \pi / 2)$ such that $g\left(\theta_{0}\right)>0$, which implies the following:

(a) $\Im\left(\sqrt{|r|} e^{i\left(2 \pi-\theta_{0}\right)}+a\right)-\Im\left(\sqrt{|r|} e^{i\left(\pi+\theta_{0}\right)}+b\right)=0$,

(b) $\Re\left(\sqrt{|r|} e^{i\left(2 \pi-\theta_{0}\right)}+a\right)-\Re\left(\sqrt{|r|} e^{i\left(\pi+\theta_{0}\right)}+b\right)>0$,

(c) $\left(\sqrt{|r|} e^{i\left(2 \pi-\theta_{0}\right)}+a\right),\left(\sqrt{|r|} e^{i\left(\pi+\theta_{0}\right)}+b\right) \in H^{-}$.

By (a), (b) and (c),

$$
\operatorname{Arg}\left(\sqrt{|r|} e^{i\left(2 \pi-\theta_{0}\right)}+a\right)-\operatorname{Arg}\left(\sqrt{|r|} e^{i\left(\pi+\theta_{0}\right)}+b\right)>0 .
$$

Define the function $h:[1, \infty) \rightarrow \mathbb{R}$ by

$$
h(k):=\operatorname{Arg}\left(k \sqrt{|r|} e^{i\left(2 \pi-\theta_{0}\right)}+a\right)-\operatorname{Arg}\left(\frac{\sqrt{|r|}}{k} e^{i\left(\pi+\theta_{0}\right)}+b\right) .
$$

The function $h$ is real valued, and $h(1)>0$. Thus, by continuity, there exists $k_{0}>1$ such that $h\left(k_{0}\right)$ remains positive,

$$
\operatorname{Arg}\left(k_{0} \sqrt{|r|} e^{i\left(2 \pi-\theta_{0}\right)}+a\right)-\operatorname{Arg}\left(\frac{\sqrt{|r|}}{k_{0}} e^{i\left(\pi+\theta_{0}\right)}+b\right)>0,
$$

but the imaginary parts differ,

$$
\Im\left(k_{0} \sqrt{|r|} e^{i\left(2 \pi-\theta_{0}\right)}+a\right)<\Im\left(\frac{\sqrt{|r|}}{k_{0}} e^{i\left(\pi+\theta_{0}\right)}+b\right) .
$$


Let

$$
A=k_{0} \sqrt{|r|} e^{i\left(2 \pi-\theta_{0}\right)}+a,
$$

and

$$
B=\frac{\sqrt{|r|}}{k_{0}} e^{i\left(\pi+\theta_{0}\right)}+b .
$$

Then (2.33) and (2.34) satisfies items (i), (ii) and (iii) of Lemma 2.5; hence, there exist $x, w \in H^{+}$such that $\left(x+r_{1}\right) w=A$ and $\left(x+r_{2}\right) w=$ $B$. Thus,

$$
\begin{aligned}
f(x, w) & =\left(\left(x+r_{1}\right) w-a\right)\left(\left(x+r_{2}\right) w-b\right) \\
& =\left(k_{0} \sqrt{|r|} e^{i\left(2 \pi-\theta_{0}\right)}\right)\left(\frac{\sqrt{|r|}}{k_{0}} e^{i\left(\pi+\theta_{0}\right)}\right)=-|r|=r .
\end{aligned}
$$

Case 3. We consider $r \in(a b, \infty)$. Since $r>a b, r=a^{\prime} b^{\prime}$, for some $a^{\prime}>a$ and $b^{\prime}>b$. Define the function $g:[\pi / 2, \pi] \rightarrow\left[a-a^{\prime}, a\right] \times\left[b-b^{\prime}, b\right]$ by

$$
g(\theta):=\left(\Re\left(a^{\prime} e^{-i \theta}\right)+a, \Re\left(b^{\prime} e^{i \theta}\right)+b\right) .
$$

Since $a-a^{\prime}, b-b^{\prime}<0, g(\pi)=\left(a-a^{\prime}, b-b^{\prime}\right)$ has negative coordinates. By continuity, there exists $\theta_{0} \in(\pi / 2, \pi)$ such that $g\left(\theta_{0}\right)$ has negative coordinates, which implies that $a^{\prime} e^{-i \theta_{0}}+a$ is in quadrant three, and $b^{\prime} e^{i \theta_{0}}+b$ is in quadrant two. Let

$$
A=a^{\prime} e^{-i \theta_{0}}+a \text { and } B=b^{\prime} e^{i \theta_{0}}+b .
$$

Again, by Lemma 2.5, there exist $x, w \in H^{+}$such that $\left(x+r_{1}\right) w=A$, and $\left(x+r_{2}\right) w=B$. Thus,

$$
f(x, w)=\left(\left(x+r_{1}\right) w-a\right)\left(\left(x+r_{2}\right) w-b\right)
$$

$$
=\left(a^{\prime} e^{-\theta_{0} i}\right)\left(b^{\prime} e^{\theta_{0} i}\right)=a^{\prime} b^{\prime}=r .
$$

To prove sufficiency, first consider $r \in(0, a b]$. By way of contradiction, assume there exist $x, w \in H^{+}$such that $\left(\left(x+r_{1}\right) w-a\right)((x+$ $\left.\left.r_{2}\right) w-b\right)=r$. Let $A=\left(\left(x+r_{1}\right) w-a\right)$ and $B=\left(\left(x+r_{2}\right) w-b\right)$. Since $x+r_{1}, x+r_{2} \in H^{+}$, the rotation by $\operatorname{Arg}(w) \in(0, \pi)$ and the shifts to the left by $a, b>0$ restrict the location of $A$ and $B$ considerably. 
Indeed, since $A B$ is a positive real number, $\operatorname{Arg}(A)+\operatorname{Arg}(B)=2 \pi$. In particular, since $r_{1}<r_{2}, B$ must be in $H^{+}$, which implies

$$
0<\operatorname{Arg}(w)<\operatorname{Arg}\left(\left(x+r_{2}\right) w\right)<\operatorname{Arg}\left(\left(x+r_{2}\right) w-b\right)<\pi
$$

and $A$ must be in $H^{-}$, which implies

(2.41) $\pi<\operatorname{Arg}\left(\left(x+r_{1}\right) w-a\right)<\operatorname{Arg}\left(\left(x+r_{1}\right) w\right)<\pi-\operatorname{Arg}(w)<2 \pi$.

The following figure illustrates inequalities (2.40) and (2.41).

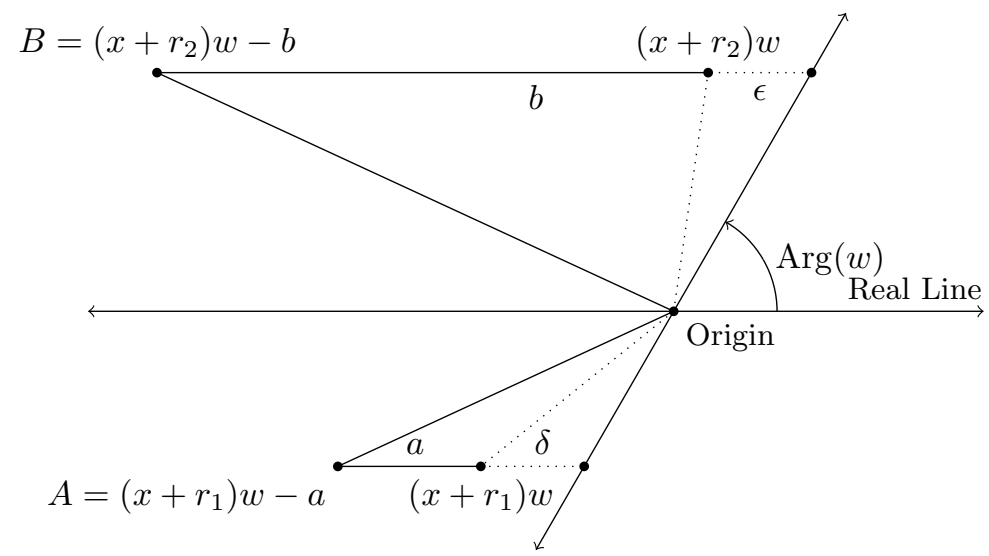

We let $\epsilon$ and $\delta$ be the horizontal distance from $\left(x+r_{1}\right) w$ and $\left(x+r_{2}\right) w$ to the line formed by $\operatorname{Arg}(w)$. In fact, $\delta=\Im\left(x+r_{1}\right) / \sin (\operatorname{Arg}(w))$, and $\epsilon=\Im\left(x+r_{2}\right) / \sin (\operatorname{Arg}(w))$, so that $\delta=\epsilon>0$. We redraw the picture 
with different labels and examine the points geometrically.

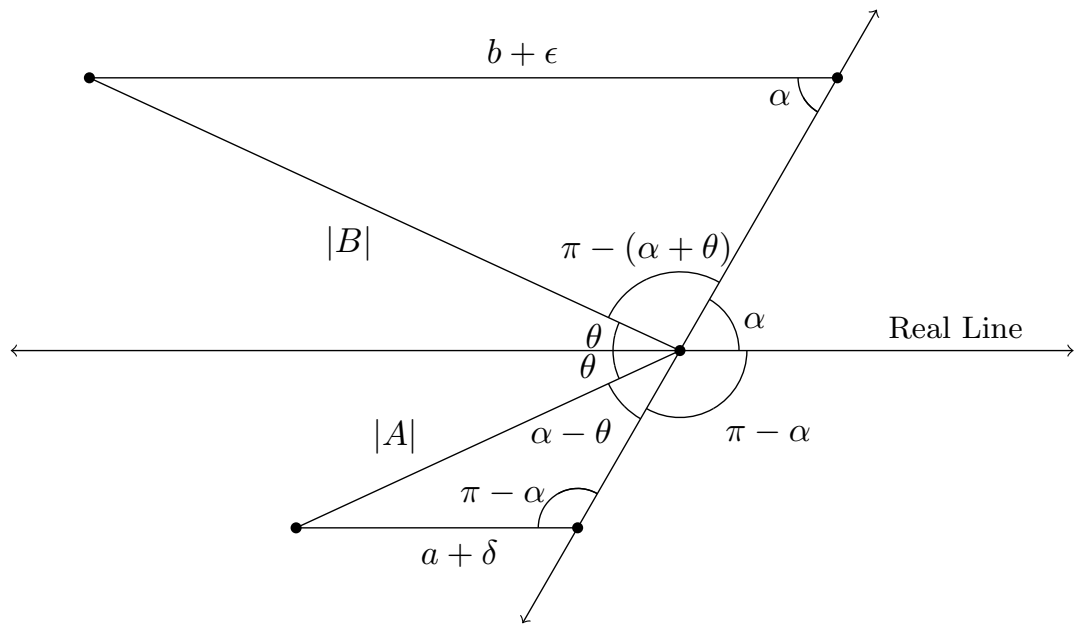

The inequalities $\alpha-\theta>0$ and $\pi-(\alpha+\theta)>0$ imply $0<\theta<\alpha<$ $\pi-\theta<\pi$, so that

$$
\sin (\theta)<\sin (\alpha)
$$

since $\sin (\theta)=\sin (\pi-\theta)$. Thus,

$$
0<\left(\frac{\sin (\theta)}{\sin (\alpha)}\right)^{2}<1,
$$

and the law of sines yields that

$$
\begin{aligned}
(a+\delta)(b+\epsilon) & =\frac{|A| \sin (\alpha-\theta)}{\sin (\pi-\alpha)} \cdot \frac{|B| \sin (\pi-(\alpha+\theta))}{\sin (\alpha)} \\
& =\left(1-\left(\frac{\sin (\theta)}{\sin (\alpha)}\right)^{2}\right)|A B|<|A B|
\end{aligned}
$$

Hence, we have the contradiction that

$$
a b<(a+\delta)(b+\epsilon)<|A B|=r .
$$

To finish the proof, consider $r=0$. By way of contradiction, suppose there are $x, w \in H^{+}$such that

$$
\left(\left(x+r_{1}\right) w-a\right)\left(\left(x+r_{2}\right) w-b\right)=0 .
$$


Thus, $\left(x+r_{1}\right) w=a$ or $\left(x+r_{2}\right) w=b$. However, neither of these can hold, since the product of any two complex numbers in $H^{+}$cannot be a non-negative real number.

Theorem 2.7. Let $a, b \geq 0, r_{1}, r_{2}, r \in \mathbb{R}$ and $r_{1} \neq r_{2}$. Then,

$$
r \in[0, a b]
$$

if and only if

$$
T:=\left(x+r_{1}\right)\left(x+r_{2}\right) D^{2}+\left(b\left(x+r_{1}\right)+a\left(x+r_{2}\right)\right) D+r
$$

(where $D:=d / d x)$, is hyperbolicity preserving.

Proof. To prove necessity, assume $r \in[0, a b]$. By Theorem 2.4, it suffices to show, for every $x, w \in H^{+}$,

$$
\left(x+r_{1}\right)\left(x+r_{2}\right) w^{2}-\left(b\left(x+r_{1}\right)+a\left(x+r_{2}\right)\right) w+r \neq 0 .
$$

By Lemma 2.6, since $a b-r \in[0, a b]$, then for every $x, w \in H^{+}$,

$$
\left(\left(x+r_{1}\right) w-a\right)\left(\left(x+r_{2}\right) w-b\right) \neq a b-r .
$$

We rewrite equation (2.46) and attain (2.45).

To prove sufficiency, suppose $T$ is hyperbolicity preserving. By Theorem 2.4, for every $x, w \in H^{+}$,

$$
\left(x+r_{1}\right)\left(x+r_{2}\right) w^{2}-\left(b\left(x+r_{1}\right)+a\left(x+r_{2}\right)\right) w+r \neq 0 .
$$

We factor $(2.47)$ to obtain

(2.48) $\left(\left(x+r_{1}\right) w-a\right)\left(\left(x+r_{2}\right) w-b\right) \neq a b-r, \quad$ for all $x, w \in H^{+}$,

which implies that $r \in[0, a b]$ by Lemma 2.6 .

Theorem 2.8. For $c_{i}, r_{j} \in \mathbb{R}, i=0,1,2, j=1,2,3, c_{2} \neq 0, r_{1} \neq r_{2}$, let $Q_{0}(x)=c_{0}, Q_{1}(x)=c_{1}\left(x-r_{3}\right)$ and $Q_{2}(x)=c_{2}\left(x-r_{1}\right)\left(x-r_{2}\right)$. Then

$$
0 \leq c_{1}^{2}\left(\frac{\left(r_{1}-r_{3}\right)\left(r_{3}-r_{2}\right)}{\left(r_{2}-r_{1}\right)^{2}}\right)-c_{0} c_{2},
$$

and $c_{0}, c_{1}, c_{2}$ are of the same sign if and only if

$$
T:=Q_{2}(x) D^{2}+Q_{1}(x) D+Q_{0}(x)
$$

(where $D:=d / d x)$, preserves hyperbolicity. 
Proof. To prove sufficiency, if $T$ preserves hyperbolicity, then by Lemma 1.6, $c_{i}, i=0,1,2$ are of the same sign and the zeros of $Q_{2}$ and $Q_{1}$ interlace. Since

$$
\begin{aligned}
T= & c_{2}\left(\left(x-r_{1}\right)\left(x-r_{2}\right) D^{2}+\frac{c_{1}}{c_{2}}\left(x-r_{3}\right) D+\frac{c_{0}}{c_{2}}\right) \\
= & c_{2}\left(\left(x-r_{1}\right)\left(x-r_{2}\right) D^{2}\right. \\
& \left.+\frac{c_{1}}{c_{2}}\left[\frac{\left(r_{1}-r_{3}\right)}{\left(r_{1}-r_{2}\right)}\left(x-r_{2}\right)+\frac{\left(r_{3}-r_{2}\right)}{\left(r_{1}-r_{2}\right)}\left(x-r_{1}\right)\right] D+\frac{c_{0}}{c_{2}}\right),
\end{aligned}
$$

then, by Theorem 2.7,

$$
\frac{c_{0}}{c_{2}} \in\left[0,\left(\frac{c_{1}}{c_{2}}\right)^{2} \frac{\left(r_{1}-r_{3}\right)\left(r_{3}-r_{2}\right)}{\left(r_{1}-r_{2}\right)^{2}}\right],
$$

and so

$$
0 \leq c_{1}^{2}\left(\frac{\left(r_{1}-r_{3}\right)\left(r_{3}-r_{2}\right)}{\left(r_{2}-r_{1}\right)^{2}}\right)-c_{0} c_{2}
$$

To prove necessity, suppose $c_{i}, i=0,1,2$, are of the same sign, and

$$
0 \leq c_{1}^{2}\left(\frac{\left(r_{1}-r_{3}\right)\left(r_{3}-r_{2}\right)}{\left(r_{2}-r_{1}\right)^{2}}\right)-c_{0} c_{2}
$$

Let

$$
a:=\frac{c_{1}}{c_{2}} \frac{\left(r_{1}-r_{3}\right)}{\left(r_{1}-r_{2}\right)}, \quad \text { and } \quad b:=\frac{c_{1}}{c_{2}} \frac{\left(r_{3}-r_{2}\right)}{\left(r_{1}-r_{2}\right)}
$$

By Theorem 2.7, we want to conclude that $a, b \geq 0$. To this end, if $c_{1}=0$, then $a, b \geq 0$. Suppose $c_{1} \neq 0$, and that $r_{1}<r_{2}$. Then (2.52) implies $0 \leq\left(r_{1}-r_{3}\right)\left(r_{3}-r_{2}\right)$, and we conclude that $r_{1} \leq r_{3} \leq r_{2}$ (i.e., $r_{3}<r_{1}<r_{2}$ cannot hold, since it implies $\left(r_{1}-r_{3}\right)\left(r_{3}-r_{2}\right)<0$, and also if $r_{1}<r_{2}<r_{3}$, then $\left.\left(r_{1}-r_{3}\right)\left(r_{3}-r_{2}\right)<0\right)$, and hence, $a, b \geq 0$. By symmetry, the same conclusion is true if $r_{2}<r_{1}$. Thus, by Theorem 2.7, $T$ preserves hyperbolicity.

The equality of (2.49) uses a fact established in [6, page 13, Lemma 1.20]. For the sake of completeness, we state the result.

Lemma 2.9 ([6, page 13, Lemma 1.20]). Assume that $f$ is a polynomial of degree $n$, with positive leading coefficient, and with zeros 
$\left\{a_{1}, \ldots, a_{n}\right\}$. Suppose that $g$ is a polynomial with positive leading coefficient. If $g$ has degree $n-1$, and we write

$$
g(x)=c_{1} \frac{f(x)}{x-a_{1}}+\cdots+c_{n} \frac{f(x)}{x-a_{n}},
$$

then $f$ and $g$ have interlacing zeros if and only if all $c_{i} \geq 0$ for $i=1,2, \ldots, n$.

We now remove the condition of $Q_{2}$ having distinct zeros. We begin with a lemma that is analogous to Lemma 2.6.

Lemma 2.10. Let $a, r \in \mathbb{R}, a \geq 0$. Set

$$
f(z):=z^{2}-a z+r, \quad z \in \mathbb{C} .
$$

Then

$$
\begin{gathered}
f(z) \neq 0 \quad \text { for all } z \in \mathbb{C}-[0, \infty) \\
\text { if and only if } r \in\left[0, \frac{a^{2}}{4}\right] .
\end{gathered}
$$

Proof. The zeros of $f$ are $\left(a \pm \sqrt{a^{2}-4 r}\right) / 2$. There are two cases to prove necessity.

Case 1. If $r<0$, then one of the zeros of $f$ is a negative real number; thus, there exist $z_{0} \in \mathbb{C}-[0, \infty)$ such that $f\left(z_{0}\right)=0$.

Case 2. If $r>a^{2} / 4$, then $f$ has two imaginary zeros; thus, the zeros of $f$ are in $\mathbb{C}-[0, \infty)$.

To prove sufficiency, suppose $0 \leq r \leq a^{2} / 4$. Then $f$ has two nonnegative real zeros so that $f$ never vanishes in $\mathbb{C}-[0, \infty)$.

Theorem 2.11. Let $a \geq 0, r, R \in \mathbb{R}$. Then,

$$
R \in\left[0, \frac{a^{2}}{4}\right]
$$

if and only if

$$
T:=(x+r)^{2} D^{2}+a(x+r) D+R
$$

is hyperbolicity preserving. 
Proof. To prove necessity, assume $R \in\left[0, a^{2} / 4\right]$. By Theorem 2.4, it suffices to show, for every $x, w \in H^{+}$,

$$
(x+r)^{2} w^{2}-a(x+r) w+R \neq 0 .
$$

We assume, on the contrary, that (2.53) is false for some $x, w \in H^{+}$. Let $z=(x+r) w$ in $(2.53)$, so that $z \in \mathbb{C}-[0, \infty)$, and

$$
z^{2}-a z+R=0 \text {. }
$$

This is impossible by Lemma 2.10, a contradiction.

To prove sufficiency, suppose $T$ is hyperbolicity preserving. By Theorem 2.4, for every $x, w \in H^{+}$,

$$
(x+r)^{2} w^{2}-a(x+r) w+R \neq 0 .
$$

Let $z=(x+r) w$ in $(2.55)$, so that $z \in \mathbb{C}-[0, \infty)$, and

$$
z^{2}-a z+R \neq 0, \quad \text { for all } z \in \mathbb{C}-[0, \infty),
$$

which, by Lemma 2.10, implies that $R \in\left[0, a^{2} / 4\right]$.

The analogous statement of Theorem 2.8 is the following, and its proof follows, mutatis mutandis, from the proof of Theorem 2.8.

Theorem 2.12. For $r, c_{i} \in \mathbb{R}, i=0,1,2, c_{2} \neq 0$, let $Q_{0}(x)=c_{0}$, $Q_{1}(x)=c_{1}(x-r)$ and $Q_{2}(x)=c_{2}(x-r)^{2}$. Then

$$
0 \leq c_{1}^{2}\left(\frac{1}{4}\right)-c_{0} c_{2}
$$

and $c_{0}, c_{1}, c_{2}$ are of the same sign, if and only if

$$
T=Q_{2}(x) D^{2}+Q_{1}(x) D+Q_{0}(x)
$$

preserves hyperbolicity.

We now wish to find a condition that combines the statements of Theorem 2.12 and Theorem 2.8. To this end, we prove the following lemma.

Lemma 2.13. For $c_{i}, r_{j} \in \mathbb{R}, i=0,1,2, j=1,2,3, c_{2} \neq 0$, let $Q_{0}(x)=c_{0}, Q_{1}(x)=c_{1}\left(x-r_{3}\right)$ and $Q_{2}(x)=c_{2}\left(x-r_{1}\right)\left(x-r_{2}\right)$. If 
$T=Q_{2}(x) D^{2}+Q_{1}(x) D+Q_{0}(x)$ is hyperbolicity preserving, then

$$
0 \leq c_{1}^{2}\left(\frac{1}{4}\right)-c_{0} c_{2}
$$

Furthermore, if $r_{1} \neq r_{2}$, then

$$
0 \leq c_{1}^{2} \frac{\left(r_{1}-r_{3}\right)\left(r_{3}-r_{2}\right)}{\left(r_{2}-r_{1}\right)^{2}}-c_{0} c_{2} \leq c_{1}^{2}\left(\frac{1}{4}\right)-c_{0} c_{2} .
$$

Thus, if $c_{1}^{2}-4 c_{0} c_{2}=0$, then $2 r_{3}=r_{1}+r_{2}$.

Proof. Theorem 2.12 deals with the case of when $r_{1}=r_{2}$, thus it suffices to show

$$
0 \leq \frac{\left(r_{1}-r_{3}\right)\left(r_{3}-r_{2}\right)}{\left(r_{2}-r_{1}\right)^{2}} \leq \frac{1}{4}
$$

The left inequality holds because $Q_{2}$ and $Q_{1}$ have interlacing zeros by Lemma 1.6. To show the right inequality, we proceed as follows,

$$
\begin{gathered}
0 \leq\left(2 r_{3}-\left(r_{1}+r_{2}\right)\right)^{2}, \\
4\left(r_{1} r_{3}+r_{2} r_{3}\right) \leq\left(r_{2}+r_{1}\right)^{2}+4 r_{3}^{2}, \\
4\left(r_{1} r_{3}-r_{1} r_{2}-r_{3}^{2}+r_{2} r_{3}\right) \leq r_{2}^{2}-2 r_{1} r_{2}+r_{1}^{2}, \\
4\left(r_{1}-r_{3}\right)\left(r_{3}-r_{2}\right) \leq\left(r_{2}-r_{1}\right)^{2} .
\end{gathered}
$$

Theorem 2.14. For $c_{i}, r_{j} \in \mathbb{R}, i=0,1,2, j=1,2,3, c_{2} \neq 0$, let $Q_{0}(x)=c_{0}, Q_{1}(x)=c_{1}\left(x-r_{3}\right)$ and $Q_{2}(x)=c_{2}\left(x-r_{1}\right)\left(x-r_{2}\right)$ with $Q_{0}(x) \ll Q_{1}(x)$ and $Q_{1}(x) \ll Q_{2}(x)$. Then

$$
T=Q_{2}(x) D^{2}+Q_{1}(x) D+Q_{0}(x)
$$

preserves hyperbolicity if and only if

$$
W\left[Q_{0}, Q_{2}\right]^{2}-W\left[Q_{0}, Q_{1}\right] W\left[Q_{1}, Q_{2}\right] \leq 0 .
$$


Proof. Since $Q_{0} \ll Q_{1}$ and $Q_{1} \ll Q_{2}$, the signs of $c_{0}, c_{1}, c_{2}$ are same, and $r_{1} \leq r_{3} \leq r_{2}$. Define

$$
\begin{aligned}
w(x):= & W\left[Q_{0}, Q_{2}\right]^{2}-W\left[Q_{0}, Q_{1}\right] W\left[Q_{1}, Q_{2}\right] \\
= & c_{0} c_{2}\left(4 c_{0} c_{2}-c_{1}^{2}\right) x^{2}+2 c_{0} c_{2}\left(-2 c_{0} c_{2}\left(r_{1}+r_{2}\right)+c_{1}^{2} r_{3}\right) x \\
& +c_{0} c_{2}\left(c_{0} c_{2}\left(r_{1}+r_{2}\right)^{2}+c_{1}^{2}\left(r_{1} r_{2}-r_{1} r_{3}-r_{2} r_{3}\right)\right) .
\end{aligned}
$$

Suppose $r_{1}=r_{2}$. Then $w(x)=-c_{0} c_{2}\left(c_{1}^{2}-4 c_{0} c_{2}\right)\left(x-r_{1}\right)^{2}$. It is clear that $w(x) \leq 0$ if and only if $0 \leq c_{1}^{2}-4 c_{0} c_{2}$; thus, we apply Theorem 2.12.

Suppose $0=c_{1}^{2}-4 c_{0} c_{2}$ and $r_{1} \neq r_{2}$. By Lemma 2.13, Theorem 2.8 can restated as, " $T$ is hyperbolicity preserving if and only if $2 r_{3}=$ $r_{1}+r_{2}$." We recalculate $w$, under the assumption that $c_{1}^{2}-4 c_{0} c_{2}=0$,

$$
\begin{aligned}
w(x)= & 4 c_{0}^{2} c_{2}^{2}\left(2 r_{3}-r_{1}-r_{2}\right) x \\
& +c_{0}^{2} c_{2}^{2}\left(2\left(r_{1}+r_{2}\right)\left(r_{1}+r_{2}-2 r_{3}\right)-\left(r_{1}-r_{2}\right)^{2}\right) .
\end{aligned}
$$

We now see that $w(x) \leq 0$, if and only if $2 r_{3}=r_{1}+r_{2}$.

Thus, we may assume $0 \neq c_{1}^{2}-4 c_{0} c_{2}$ and $r_{1} \neq r_{2}$, in which case the graph of $w(x)$ is a parabola with vertex

$$
\left(r_{3}, \frac{c_{0} c_{1}^{2} c_{2}}{c_{1}^{2}-4 c_{0} c_{2}}\left(c_{0} c_{2}\left(r_{1}-r_{2}\right)^{2}+c_{1}^{2}\left(r_{1}-r_{3}\right)\left(r_{2}-r_{3}\right)\right)\right) .
$$

Since $w$ is a quadratic, $w(x) \leq 0$ if and only if the leading coefficient

$$
c_{0} c_{2}\left(4 c_{0} c_{1}-c_{1}^{2}\right)<0,
$$

and $y$-coordinate of the vertex

$$
\frac{c_{0} c_{1}^{2} c_{2}}{c_{1}^{2}-4 c_{0} c_{2}}\left(c_{0} c_{2}\left(r_{1}-r_{2}\right)^{2}+c_{1}^{2}\left(r_{1}-r_{3}\right)\left(r_{2}-r_{3}\right)\right) \leq 0 .
$$

Thus, we can say that $w(x) \leq 0$ if and only if $0<c_{1}^{2}-4 c_{0} c_{1}$ and $0 \leq$ $c_{1}^{2}\left(r_{1}-r_{3}\right)\left(r_{3}-r_{2}\right)-c_{0} c_{2}\left(r_{1}-r_{2}\right)^{2}$. By Lemma 2.13 and Theorem 2.8, those conditions are equivalent to $T$ preserving hyperbolicity.

It is unnecessary to assume that the polynomial coefficients of $T$ have real zeros as this will follow from Lemma 1.6. Furthermore, if $Q_{2}$ 
is a quadratic, then Lemma 1.6 states that $Q_{1}$ cannot be a non-zero constant, if $T$ is to preserve hyperbolicity. To summarize, we restate Theorem 2.14 with a little more generality.

Theorem 1.7. Suppose $Q_{2}, Q_{1}, Q_{0}$ are real polynomials such that $\operatorname{deg}\left(Q_{2}\right)=2, \operatorname{deg}\left(Q_{1}\right) \leq 1$ and $\operatorname{deg}\left(Q_{0}\right)=0$. Then

$$
T=Q_{2} D^{2}+Q_{1} D+Q_{0}
$$

preserves hyperbolicity if and only if

$$
W\left[Q_{0}, Q_{2}\right]^{2}-W\left[Q_{0}, Q_{1}\right] W\left[Q_{1}, Q_{2}\right] \leq 0, \quad \text { and } \quad Q_{0} \ll Q_{1} \ll Q_{2} .
$$

3. Multiplier sequences. We now wish to establish several consequences of the above quadratic operators.

Definition 3.1. Let $\left\{P_{n}\right\}$ be a basis for $\mathbb{R}[x]$. We call a set of polynomials simple if each polynomial $P_{n}$ has degree $n$. Let $\left\{A_{n}\right\}$ be a sequence of real numbers. If there is a linear operator, $T$, such that $T\left[P_{n}\right]=A_{n} P_{n}$ for every $n \in \mathbb{N}$, then we call $T$ a $P_{n}$-multiplier operator. If there is a hyperbolicity preserver, $T$, such that $T\left[P_{n}\right]=A_{n} P_{n}$ for every $n \in \mathbb{N}$, then we call $T$ a $P_{n}$-multiplier sequence.

The following theorem summarizes the natural relationship between differential equations, differential operators and $P_{n}$-multiplier sequences.

Theorem 3.2. Let $P_{n}$ be a simple basis for $\mathbb{R}[x]$. Suppose, for each $n \in \mathbb{N}, P_{n}$ satisfies the differential equation

$$
\sum_{k=0}^{\infty} Q_{k}(x) y^{(k)}=A_{n} y,
$$

where $\left\{Q_{k}\right\}$ is a sequence of real polynomials and $\left\{A_{n}\right\}$ is a sequence of real numbers. Then $A_{n}$ is a $P_{n}$-multiplier sequence if and only if

$$
\sum_{k=0}^{\infty} Q_{k} D^{k}
$$

is a hyperbolicity preserver. 
Using Theorem 2.8, we can restate Theorem 3.2.

Theorem 3.3. Let $P_{n}$ be a simple set for $\mathbb{R}[x]$, and let $\left\{A_{n}\right\}$ be a sequence of real numbers. Let $c_{i}, r_{j} \in \mathbb{R}, i=0,1,2, j=1,2,3$ and $c_{2} \neq 0$. Suppose, for each $n \in \mathbb{N}$, that $P_{n}$ satisfies the differential equation

$$
c_{2}\left(x-r_{1}\right)\left(x-r_{2}\right) y^{\prime \prime}+c_{1}\left(x-r_{3}\right) y^{\prime}+c_{0} y=A_{n} y .
$$

Then $\left\{A_{n}\right\}$ is a $P_{n}$-multiplier sequence if, and only if, $c_{0}, c_{1}, c_{2}$ are of the same sign and

$$
0 \leq c_{1}^{2}\left(\frac{\left(r_{1}-r_{3}\right)\left(r_{3}-r_{2}\right)}{\left(r_{2}-r_{1}\right)^{2}}\right)-c_{2} c_{0} .
$$

In light of Theorem 2.12, we take $\left[\left(r_{1}-r_{3}\right)\left(r_{3}-r_{2}\right)\right] /\left(r_{2}-r_{1}\right)^{2}=1 / 4$ in the case that $r_{1}=r_{2}=r_{3}$. Also, if $r_{1}=r_{2}$ and $r_{1} \neq r_{3}$, then $\left\{A_{n}\right\}$ is not a $P_{n}$-multiplier sequence.

A large number of very well-known bases for $\mathbb{R}[x]$ satisfy differential equations of the above form ([12, pages $173,188,204,258])$. We exhibit classes of multiplier sequences for Legendre, Jacobi and the standard basis. We state the corresponding differential equations:

Standard basis:

$$
A x^{2}\left(x^{n}\right)^{\prime \prime}+B x\left(x^{n}\right)^{\prime}+C\left(x^{n}\right)=(A n(n-1)+B n+C) x^{n} .
$$

Lengendre polynomials:

$$
A\left(x^{2}-1\right) P_{n}^{\prime \prime}+2 A x P_{n}^{\prime}+B P_{n}=(A n(n+1)+B) P_{n} .
$$

Jacobi polynomials:

$$
\begin{aligned}
& A\left(x^{2}-1\right)\left(P_{n}^{(\alpha, \beta)}\right)^{\prime \prime} \\
& \begin{array}{r}
+A((\alpha+\beta+2) x-(\beta-\alpha))\left(P_{n}^{(\alpha, \beta)}\right)^{\prime}+B P_{n}^{(\alpha, \beta)} \\
\quad=(A n(n+\alpha+\beta+1)+B) P_{n}^{(\alpha, \beta)}
\end{array}
\end{aligned}
$$

We now establish several classes of multiplier sequences. Note that multiplier sequences that arise from the standard basis are commonly referred to as classic multiplier sequences. 
Theorem 3.4. Let $A, B, C \in \mathbb{R}$. Then $\{A n(n-1)+B n+C\}$ is a classic multiplier sequence if and only if $A, B, C$ are of the same sign and

$$
0 \leq B^{2}-4 A C \text {. }
$$

Theorem 3.5 ([1, Theorem 11]). Let $A, B \in \mathbb{R}, A \neq 0$. Then $\{A n(n+1)+B\}$ is a $P_{n}$-multiplier sequence (Lengendre multiplier sequence) if and only if

$$
0 \leq \frac{B}{A} \leq 1
$$

Theorem 3.6. Let $A, B \in \mathbb{R}, A \neq 0$. Then $\{A n(n+\alpha+\beta+1)+B\}$ is a $P_{n}^{(\alpha, \beta)}$-multiplier sequence (Jacobi multiplier sequence, i.e., $-1<\alpha, \beta$ ) if and only if

$$
0 \leq \frac{B}{A} \leq(\alpha+1)(\beta+1) .
$$

Proof. We note that

$$
A, A(\alpha+\beta+2) \text { and } B \text { have the same sign }
$$

and

$$
(A(\alpha+\beta+2))^{2}\left(1-\frac{\beta-\alpha}{\alpha+\beta+2}\right)\left(1+\frac{\beta-\alpha}{\alpha+\beta+2}\right)-4 A B \geq 0
$$

is equivalent to

$$
0 \leq \frac{B}{A} \leq(\alpha+1)(\beta+1)
$$

\section{REFERENCES}

1. K. Blakeman, E. Davis, T. Forgács, and K. Urabe, On Legendre multiplier sequences, arXiv:1108.4662.

2. J. Borcea and P. Brändén, Pólya-Schur master theorems for circular domains and their boundaries, Ann. Math. 170 (2009), 465-492.

3. , Multivariate Pólya-Schur classification problems in the Weyl algebra, Proc. Lond. Math. Soc. 101 (2010), 73-104.

4. P. Brändén, A generalization of the Heine-Stieltjes theorem, Constr. Approx. 34 (2011), 135-148.

5. T. Craven and G. Csordas, Composition theorems, multiplier sequences and complex zero decreasing sequences, Adv. Compl. Anal. Appl. 3, Kluwer Academic Publishers, Boston, MA, 2004. 
6. S. Fisk, Polynomials, roots, and interlacing, arXiv:0612833v2.

7. T. Forgács and A. Pitrowski, Multiplier sequences for generalized Laguerre bases, arXiv:1002.0759.

8. J. Peetre, Une caractérisation abstraite des opérateurs différentiels, Math. Scand. 7 (1959), 211-218; Erratum, ibid. 8 (1960), 116-120.

9. A. Piotrowski, Linear operators and the distribution of zeros of entire functions, Ph.D. Dissertation, University of Hawaii, Manoa, May 2007.

10. G. Pólya and J. Schur, Über zwei Arten von Faktorenfolgen in der Theorie der algebraischen Gleichungen, J. reine angew. Math. 144 (1914), 89-113.

11. Q.I. Rahman and G. Schmeisser, Analytic theory of polynomials, Oxford University Press, Oxford, 2002.

12. E. Rainville, Special functions, Chelsea, New York, 1960.

326 BALdwin RD, \#251, Odenton, MD 21113

Email address: rdbates@math.hawaii.edu

University of Hawail at ManoA, 2500 Campus Road, Honolulu, Hi 96822

Email address: yoshi@math.hawaii.edu 